\title{
BIBLIOGRAPHY
}

1. S. P. Diliberto, On systems of ordinary differential equations, Contributions to the Theory of Nonlinear Oscillations, Annals of Mathematics Studies, no. 20, Princeton, 1950, pp. 1-38.

2. - A note on linear ordinary differential equations, Proc. Amer. Math. Soc. vol. 8 (1957) pp. 462-464. 473.

3. O. Perron, Über eine Matrixtransformation, Math. Zeit. vol. 32 (1930) pp. 465-

4. W. T. Reid, Some results on the growth of solutions of differential systems, Bull. Amer. Math. Soc. Abstract 48-11-319.

NORTHWESTERN UNIVERSITY

\section{AN IRREDUCIBLE UNITARY REPRESENTATION OF A COMPACT GROUP IS FINITE DIMENSIONAL}

\author{
PAUL KOOSIS
}

This note contains a proof of the statement included in the title. The result is certainly known; Anna Hurevitsch [1] proves it using the theorem of Peter and Weyl, under the assumption that the group satisfies the second axiom of countability. It would perhaps nevertheless still be desirable to have at hand a short proof which uses the minimum amount of machinery, then the Peter-Weyl theorem, as well as the rest of the representation theory for compact groups [2, Ch. IV] would follow directly from the classical work of Gelfand and Raikov [3] on the unitary Hilbert space representations of a general locally compact group. Since Cartan and Godement [4] have already shown how the theory of abelian locally compact groups follows from the general one of Gelfand and Raikov, one would have available a rather unified treatment of the two branches of topological group theory which have been most extensively studied, a unity which is not so apparent in existing expositions of the subject (cf. [5]).

Such a proof is given here. It was worked out some time ago, in 1950 , but its publication, put off until now, is maybe yet worth while in view of the above considerations.

(The referee has called my attention to a proof similar to the one given here, save that the condition of complete continuity used below is replaced by that of being an operator of Hilbert-Schmidt type. It

Received by the editors September 5, 1956. 
is found in a set of mimeographed notes [9, p. 22-107], published some years after 1950 , and of which I was unaware.)

Let $G$ be a compact topological group, with identity $e$. Integration over $G$ with respect to Haar measure shall be donated by $\int \cdots d t$, the Haar measure being supposed so normalized that $\int d t=1$.

Suppose we are given a unitary representation of $G$. This means that we have a Hilbert space $H$ and for each $t \in G$ a unitary operator $V_{t}$ on $H$, these operators satisfying the conditions:

(i) $V_{s} V_{t}=V_{s t}$ for $s, t \in G$ and $V_{e}=1$.

(ii) For given $x$ and $y \in H,\left(V_{t} x, y\right)$ is a continuous function of $t$ on $G$.

Let us choose an arbitrary nonzero $x_{0} \in H$; this element shall be fixed throughout the remainder of the discussion. If $f$ is any continuous function on $G$, it is evident that the integral $\int f(t) V_{t} x_{0} d t$ exists in the ordinary sense (as the limit of sums of elements of $H$ ) and defines a unique element of $H$. (See $[6, \S 3.5]$, for a detailed discussion of such integrals.) It is plain that this process of integration has linearity and associativity properties analogous to those possessed by the operation of taking finite linear combinations of elements of $H$, and generates a continuous mapping from the space of continuous functions on $G$ into $H$.

We define a linear operator $T$ on $H$ by putting

$$
T y=\int\left(y, V_{t} x_{0}\right) V_{t} x_{0} d t \text { for } y \in H,
$$

and establish a series of statements about $T$.

1. $T$ is bounded and self-adjoint. We have, in obvious analogy to the case of finite sums:

$$
\|T y\| \leqq \int\left|\left(y, V_{t} x_{0}\right)\right|\left\|V_{\imath} x_{0}\right\| d t \leqq \int\|y\|\left\|V_{t} x_{0}\right\|^{2} d t=\|y\|\left\|x_{0}\right\|^{2},
$$

since the $V_{t}$ are unitary.

Also, for $y$ and $z \in H,(T y, z)=\int\left(y, V_{t} x_{0}\right)\left(V_{t} x_{0}, z\right) d t$, whilst ${ }^{1}(y, T z)$ $=\int\left(z, V_{t} x_{0}\right) *\left(y, V_{t} x_{0}\right) d t=\int\left(y, V_{t} x_{0}\right)\left(V_{t} x_{0}, z\right) d t=(T y, z)$.

2. $T \neq 0$. $\left(V_{e} x_{0}, x_{0}\right)=\left\|x_{0}\right\|^{2}>0$, wherefore by (ii), $\left(V_{t} x_{0}, x_{0}\right)$ is bounded away from zero on some neighborhood of $e$ having positive Haar measure. Therefore,

$$
\left(T x_{0}, x_{0}\right)=\int\left(x_{0}, V_{t} x_{0}\right)\left(V_{t} x_{0}, x_{0}\right) d t=\int\left|\left(V_{t} x_{0}, x_{0}\right)\right|^{2} d t>0 .
$$

Because $T$ is self-adjoint, we then have:

$1 c^{*}$ denotes the complex conjugate of the complex number $c$. 
3. $T^{2} \neq 0$.

4. For $s \in G, V_{s} T=T V_{s}$. Given $y \in H, V_{s} T y=\int\left(y, V_{t} x_{0}\right) V_{s} V_{t} x_{0} d t$. On replacing $t$ by $s^{-1} t$, and using (i) together with the invariance of Haar measure, we see that this last integral becomes:

$$
\int\left(y, V_{s}^{-1} V_{t} x_{0}\right) V_{t} x_{0} d t=\int\left(V_{s} y, V_{t} x_{0}\right) V_{t} x_{0} d t=T V_{s} y,
$$

since $V_{s}$ is unitary.

5. $T^{2}$ is completely continuous. If $S$ denotes the unit sphere in $H$, we shall show that $T^{2} S$ is totally bounded. I claim first that the functions on $G$ :

$$
f_{y}(s)=\left(T y, V_{s} x_{0}\right)
$$

form an equicontinuous and equibounded set as $y$ ranges over $S$. We have, for $r$ and $s \in G$,

$$
\begin{aligned}
\left|f_{y}(r)-f_{y}(s)\right| & =\left|\int\left(y, V_{t} x_{0}\right)\left\{\left(V_{t} x_{0}, V_{r} x_{0}\right)-\left(V_{t} x_{0}, V_{s} x_{0}\right)\right\} d t\right| \\
& \leqq\left\|y \left|\left\|\left|\left\|x_{0}\right\| \sup _{t \in G}\right|\left(V_{t}^{-1} V_{r} x_{0}, x_{0}\right)-\left(V_{t}^{-1} V_{s} x_{0}, x_{0}\right) \mid .\right.\right.\right.
\end{aligned}
$$

The supremum on the right will, by (ii), be small if $r$ and $s$ are sufficiently close, since continuous functions on the compact group $G$ are in fact uniformly continuous. This demonstrates the asserted equicontinuity; equiboundedness is now clear.

It follows by Ascoli's theorem that the set $\left\{f_{y} ; y \in S\right\}$ of functions on $G$ is totally bounded. $T^{2} S=\left\{\int f_{y}(t) V_{a} x_{0} d t ; y \in S\right\}$ is then totally bounded in $H$, by the remarks immediately preceding the introduction of the transformation $T$.

We are now prepared to show:

Theorem. An irreducible unitary representation of a compact group is finite dimensional.

Proof. Irreducibility of the given unitary representation means, with continuation of the above notation, that $H$ has no proper projection which commutes simultaneously with all the $V_{t}, t \in G$. We wish to show that $H$ is finite dimensional.

The operator $T^{2}$ is, by 1 ., self-adjoint and bounded, hence has a spectral decomposition

$$
T^{2}=\int_{0}^{m} u d E_{u} \text {, the } E_{u} \text { being projections on } H \text {. }
$$


By 4., $V_{s} T^{2}=T^{2} V_{s}$ for $s \in G$, hence also every $E_{u}$ commutes with the $V_{8}[7$, p. 25]; this, together with the hypothesized irreducibility, implies that every $E_{u}$ is 0 or 1 . It follows that $T^{2}=a \cdot 1, a$ constant, moreover, $a$ cannot be zero by 3 .

Finally, $T^{2} S=a S$ is, in accordance with 5 ., totally bounded, and since $a \neq 0$ it follows by a well-known theorem of Mazur [8, p. 84] that $H$ is finite dimensional.

This paper is a revision of a report prepared for Professor J. L. Kelley's seminar on topological groups at the University of California during the Spring of 1950; I wish to acknowledge his advice and assistance.

\section{REFERENCES}

1. A. Hurevitsch, Unitary representations in Hilbert space of a compact topological group, Rec. Math. (Mat. Sbornik) N.S. T13 (55): 1 (1943) pp. 79-86.

2. L. Pontrjagin, Topological groups, Princeton University Press, 1946.

3. I. M. Gel'fand, and D. A. Raikov, Irreducible unitary representations of locally bicompact groups, Rec. Math. (Mat. Sbornik) N.S., T13 (55): 2-3 (1943) pp. 301-316 (in Russian).

4. H. Cartan, and R. Godement, Théorie de la dualité et analyse harmonique dans les groupes localement compacts, Ann. École Norm. (3) vol. 64 (1947) pp. 79-99.

5. L. H. Loomis, An introduction to abstract harmonic analysis, New York, Van Nostrand, 1953.

6. E. Hille, Functional analysis and semigroups, Amer. Math. Soc. Colloquium Publications vol. 31, 1948.

7. B. von Sz.-Nagy, Spektraldarstellung linearer Transformationen des Hilbertschen Raumes, Berlin, Springer, 1942.

8. S. Banach, Théorie des opérations linéaires, Warsaw, Monografje Mat., 1932.

9. Seminaire Sophus Lie, Ecole Normale Supérieure, Paris, 1955.

Institute of Mathematical Sciences, New York University 